\title{
Characterization of SETD1A haploinsufficiency in humans and Drosophila defines a novel neurodevelopmental syndrome
}

Kummeling, Joost ; Stremmelaar, Diante E ; Raun, Nicholas ; Reijnders, Margot R F ; Willemsen, Marjolein H ; Ruiterkamp-Versteeg, Martina ; Schepens, Marga ; Man, Calvin C O ; Gilissen, Christian ; Cho, Megan T ; McWalter, Kirsty ; Sinnema, Margje ; Wheless, James W ; Simon, Marleen E H ; Genetti, Casie A ; Casey, Alicia M ; Terhal, Paulien A ; van der Smagt, Jasper J ; van Gassen, Koen L I ; Joset, Pascal ; Bahr, Angela ; Steindl, Katharina ; Rauch, Anita ; Keller, Elmar ; Raas-Rothschild, Annick ; Koolen, David A ; Agrawal, Pankaj B ; Hoffman, Trevor L ; Powell-Hamilton, Nina N ;

Thiffault, Isabelle; et al

\begin{abstract}
Defects in histone methyltransferases (HMTs) are major contributing factors in neurodevelopmental disorders (NDDs). Heterozygous variants of SETD1A involved in histone H3 lysine 4 (H3K4) methylation were previously identified in individuals with schizophrenia. Here, we define the clinical features of the Mendelian syndrome associated with haploinsufficiency of SETD1A by investigating 15 predominantly pediatric individuals who all have de novo SETD1A variants. These individuals present with a core set of symptoms comprising global developmental delay and/or intellectual disability, subtle facial dysmorphisms, behavioral and psychiatric problems. We examined cellular phenotypes in three patient-derived lymphoblastoid cell lines with three variants: p.Gly535Alafs*12, c.458224582del AG, andp.Tyr 1499Asp.ThesepatientcelllinesdisplayedDN Adamagerepairde fectsthatwerecomparabletoprevio mediateddepletion of SET D1A.Thissuggestedthatthesevariants, includingthep.Tyr 1499 AspinthecatalyticSETdomain, of-function $(L o F)$ alleles.Previousstudiesdemonstratedarole for SET D1Aincellcyclecontrolanddif ferentiation.How
\end{abstract}

DOI: https://doi.org/10.1038/s41380-020-0725-5

Posted at the Zurich Open Repository and Archive, University of Zurich

ZORA URL: https://doi.org/10.5167/uzh-192403

Journal Article

Accepted Version

Originally published at:

Kummeling, Joost; Stremmelaar, Diante E; Raun, Nicholas; Reijnders, Margot R F; Willemsen, Marjolein H; Ruiterkamp-Versteeg, Martina; Schepens, Marga; Man, Calvin C O; Gilissen, Christian; Cho, Megan T; McWalter, Kirsty; Sinnema, Margje; Wheless, James W; Simon, Marleen E H; Genetti, Casie A; Casey, Alicia M; Terhal, Paulien A; van der Smagt, Jasper J; van Gassen, Koen L I; Joset, Pascal; Bahr, Angela; Steindl, Katharina; Rauch, Anita; Keller, Elmar; Raas-Rothschild, Annick; Koolen, David A; Agrawal, Pankaj B; Hoffman, Trevor L; Powell-Hamilton, Nina N; Thiffault, Isabelle; et al (2021). Characterization of SETD1A haploinsufficiency in humans and Drosophila defines a novel neurodevelopmental syndrome. Molecular Psychiatry, 26(6):2013-2024.

DOI: https://doi.org/10.1038/s41380-020-0725-5 


\section{UNIVERSITYOF BIRMINGHAM \\ University of Birmingham}

\section{Characterization of SETD1A haploinsufficiency in humans and Drosophila defines a novel neurodevelopmental syndrome}

Kummerling, J; Stremmelaar, D E ; Raun, N; Reijnders, M R F; Willemsen, M H; RuiterkampVersteeg, M; Schepens, M; Man, C C O; Gilissen, C; Cho, M T; McWalter, K; Sinnema, M; Wheless, J W; Simon, M E H; Genetti, C A; Casey, A M; Terhal, P A; van der Smagt, J J; van Gassen, K L I; Joset, P

DOI:

10.1038/s41380-020-0725-5

License:

Other (please specify with Rights Statement)

\section{Document Version}

Peer reviewed version

Citation for published version (Harvard):

Kummerling, J, Stremmelaar, DE, Raun, N, Reijnders, MRF, Willemsen, MH, Ruiterkamp-Versteeg, M,

Schepens, M, Man, CCO, Gilissen, C, Cho, MT, McWalter, K, Sinnema, M, Wheless, JW, Simon, MEH, Genetti, CA, Casey, AM, Terhal, PA, van der Smagt, JJ, van Gassen, KLI, Joset, P, Bahr, A, Steindl, K, Rauch, A, Keller, E, Raas-Rothschild, A, Koolen, DA, Agrawal, PB, Hoffman, TL, Powell-Hamilton, NN, Thiffault, I, Engleman, K, Zhou, D, Bodamer, O, Hoefele, J, Riedhammer, KM, Schwaibold, EMC, Tasic, V, Schubert, D, Top, D, Pfundt, R, Higgs, M, Kramer, JM \& Kleefstra, T 2020, 'Characterization of SETD1A haploinsufficiency in humans and Drosophila defines a novel neurodevelopmental syndrome', Molecular Psychiatry.

https://doi.org/10.1038/s41380-020-0725-5

Link to publication on Research at Birmingham portal

Publisher Rights Statement:

Kummeling, J., Stremmelaar, D.E., Raun, N. et al. Characterization of SETD1A haploinsufficiency in humans and Drosophila defines a novel neurodevelopmental syndrome. Mol Psychiatry (2020). https://doi.org/10.1038/s41380-020-0725-5

Subject to Springer Nature re-use terms. For further information see: https://www.nature.com/nature-research/editorial-policies/selfarchiving-and-license-to-publish\#AAMtermsV1

\section{General rights}

Unless a licence is specified above, all rights (including copyright and moral rights) in this document are retained by the authors and/or the copyright holders. The express permission of the copyright holder must be obtained for any use of this material other than for purposes permitted by law.

- Users may freely distribute the URL that is used to identify this publication.

- Users may download and/or print one copy of the publication from the University of Birmingham research portal for the purpose of private study or non-commercial research.

- User may use extracts from the document in line with the concept of 'fair dealing' under the Copyright, Designs and Patents Act 1988 (?)

- Users may not further distribute the material nor use it for the purposes of commercial gain.

Where a licence is displayed above, please note the terms and conditions of the licence govern your use of this document.

When citing, please reference the published version.

Take down policy

While the University of Birmingham exercises care and attention in making items available there are rare occasions when an item has been uploaded in error or has been deemed to be commercially or otherwise sensitive.

If you believe that this is the case for this document, please contact UBIRA@lists.bham.ac.uk providing details and we will remove access to the work immediately and investigate. 


\section{Title}

Characterization of SETD1A haploinsufficiency in humans and Drosophila defines a novel neurodevelopmental syndrome.

Author list

Joost Kummeling ${ }^{1^{*}}$

Diante E Stremmelaar ${ }^{1^{*}}$

Nicholas Raun ${ }^{2,3 *}$

Margot RF Reijnders ${ }^{4}$

Marjolein $\mathrm{H}_{\text {Willemsen }}{ }^{1}$

Martina Ruiterkamp-Versteeg ${ }^{1}$

Marga Schepens ${ }^{1}$

Calvin CO Man ${ }^{1}$

Christian Gilissen ${ }^{1}$

Megan T Cho ${ }^{5}$

Kirsty McWalter ${ }^{5}$

Margje Sinnema ${ }^{4}$

James W Wheless ${ }^{6}$

Marleen EH Simon ${ }^{7}$

Casie A Genetti ${ }^{8,9}$

Alicia M Casey ${ }^{10}$ 
Paulien A Terhal ${ }^{7}$

Jasper J van der Smagt ${ }^{7}$

Koen L van Gassen ${ }^{7}$

Pascal Joset ${ }^{11}$

Angela Bahr ${ }^{11}$

Katharina Steindl ${ }^{11}$

Anita Rauch ${ }^{11}$

Elmar Keller ${ }^{12}$

Annick Raas-Rothschild ${ }^{13}$

David A Koolen ${ }^{1}$

Pankaj B Agrawal ${ }^{8,9,14}$

Trevor L Hoffman ${ }^{15}$

Nina N Powell-Hamilton ${ }^{16}$

Isabelle Thiffault ${ }^{17}$

Kendra Engleman ${ }^{18}$

Dihong Zhou ${ }^{18}$

Olaf Bodamer ${ }^{8}$

Julia Hoefele ${ }^{19}$

Korbinian M Riedhammer ${ }^{19,20}$ 
Eva MC Schwaibold ${ }^{21}$

Velibor Tasic ${ }^{22}$

Dirk Schubert ${ }^{23}$

Deniz Top ${ }^{24}$

Rolph Pfundt ${ }^{1}$

Martin R Higgs ${ }^{25 \#}$

Jamie M Kramer ${ }^{2,3 \#}$

Tjitske Kleefstra ${ }^{1 \#}$

Affiliations

* and \#: All authors contributed equally to this work.

1. Department of Human Genetics, Donders Institute for Brain, Cognition and Behavior, Radboud university medical center, P.O. Box 9101, $6500 \mathrm{HB}$, Nijmegen, the Netherlands.

2. Department of Biochemistry and Molecular Biology, Dalhousie University, Halifax, Nova Scotia, Canada.

3. Department of Physiology and Pharmacology, The University of Western Ontario, London, Ontario, Canada.

4. Department of Clinical Genetics and School for Oncology \& Developmental Biology (GROW), Maastricht University Medical Center, Maastricht 6229 ER, the Netherlands.

5. GeneDx, Gaithersburg, MD 20877, USA. 
6. Division of Pediatric Neurology, University of Tennessee Health Science Center, Memphis, TN, United States of America. Neuroscience Institute \& Le Bonheur Comprehensive Epilepsy Program, Le Bonheur Children's Hospital, Memphis, TN, United States of America.

7. Department of Genetics, University Medical Center Utrecht, Utrecht University, Utrecht, The Netherlands.

8. Division of Genetics and Genomics, Department of Medicine, Boston Children's Hospital/Harvard Medical School, Boston, Massachusetts, USA.

9. The Manton Center for Orphan Disease Research, Boston Children's Hospital and Harvard Medical School, Boston, MA, 02115, USA.

10. Division of Pulmonary and Respiratory Diseases, Boston Children's Hospital and Harvard Medical School, Boston, MA, 02115, USA.

11. Institute of Medical Genetics, University of Zurich, Schlieren-Zurich, 8952, Switzerland.

12. Division of Neuropediatrics, Cantonal Hospital Graubuenden, Chur, Switzerland.

13. Institute of Rare Disease, Danek Gertner Institute of Human Genetics, Sheba Medical Center, Tel Hashomer, Israel; Sackler Faculty of Medicine, Tel Aviv University, Ramat Aviv, Israel.

14. Division of Newborn Medicine, Boston Children's Hospital and Harvard Medical School, Boston, MA, 02115, USA.

15. Regional Department of Genetics, Southern California Kaiser Permanente Medical Group, 1188 N. Euclid Street, Anaheim, CA 92801, USA.

16. Division of Medical Genetics, Alfred I. duPont Hospital for Children, Wilmington, DE 19803, USA.

17. Department of Pathology and Laboratory Medicine; Center for Pediatric Genomic Medicine, Children's Mercy Hospital; University of Missouri-Kansas City School of Medicine. 
18. Division of Clinical Genetics, Children's Mercy Hospital; Department of Pediatrics, Children's Mercy Hospital; University of Missouri-Kansas City School of Medicine.

19. Institute of Human Genetics, Klinikum rechts der Isar, Technical University of Munich, Munich, Germany.

20. Department of Nephrology, Klinikum rechts der Isar, Technical University of Munich, Munich, Germany.

21. Institute of Human Genetics, Heidelberg University, Heidelberg, Germany.

22. University Children's Hospital, Medical School Skopje, Skopje, North-Macedonia.

23. Department of Cognitive Neuroscience, Donders Institute for Brain, Cognition and Behaviour, Radboud University Medical Center, P.O. Box 9101, 6500 HB, Nijmegen, the Netherlands.

24. Department of Pediatrics, Dalhousie University, Halifax Nova Scotia, Canada.

25. Institute of Cancer and Genomic Sciences, College of Medical and Dental Sciences, University of Birmingham, Birmingham, B15 2TT, United Kingdom.

\section{Correspondence}

Tjitske Kleefstra (MD, PhD), Department of Human Genetics, Radboud University Medical Center, Nijmegen, PO Box 9101, 6500 HB, the Netherlands.

Telephone: 0031243613946.

E-mail: Tjitske.Kleefstra@radboudumc.nl 


\section{Abstract}

Defects in histone methyltransferases (HMTs) are major contributing factors in neurodevelopmental disorders (NDDs). Heterozygous variants of SETD1A involved in histone H3 lysine 4 (H3K4) methylation were previously identified in individuals with schizophrenia. Here, we define the clinical features of the Mendelian syndrome associated with haploinsufficiency of SETD1A by investigating 15 predominantly pediatric individuals who all have de novo SETD1A variants. These individuals present with a core set of symptoms comprising global developmental delay and/or intellectual disability, subtle facial dysmorphisms, behavioral and psychiatric problems. We examined cellular phenotypes in three patient derived lymphoblastoid cell lines with three variants: p.Gly535Alafs*12, c.4582-2_4582delAG, and p.Tyr1499Asp. These patient cell lines displayed DNA damage repair defects that were comparable to previously observed RNAi-mediated depletion of SETD1A. This suggested that these variants, including the p.Tyr1499Asp in the catalytic SET domain, behave as Loss-of-Function (LoF) alleles. Previous studies demonstrated a role for SETD1A in cell cycle control and differentiation. However, individuals with SETD1A variants do not show major structural brain defects or severe microcephaly, suggesting that defective proliferation and differentiation of neural progenitors is unlikely the single underlying cause of the disorder. We show here that the Drosophila melanogaster SETD1A orthologue is required in postmitotic neurons of the fly brain for normal memory, suggesting a role in post development neuronal function. Together, this study defines a neurodevelopmental disorder caused by dominant de novo LoF variants in SETD1A and further supports a role for H3K4 methyltransferases in the regulation of neuronal processes underlying normal cognitive functioning.

\section{Keywords}

SETD1A; histone methylation; neurodevelopmental disorder; intellectual disability; memory;

Drosophila Set1; DNA damage response 


\section{Introduction}

Neurodevelopmental disorders (NDDs) comprise a heterogeneous group of disorders including intellectual disability (ID) and autism spectrum disorders (ASDs) ${ }^{1}$. Approximately 1:200 to 1:450 children are born with a de novo pathogenic gene variant causing a Mendelian NDD ${ }^{2}$. Interestingly, recent large-scale exome sequencing studies have shown that defects in histone methyltransferases (HMTs) are major contributors to ASD and ID ${ }^{3,4}$.

One of the genes involved in epigenetic modification through histone methylation is SETD1A (also known as KMT2F), which encodes a histone methyltransferase that mediates trimethylation of histone $\mathrm{H} 3$ on lysine 4 (H3K4me3) at promoters of active genes ${ }^{5}$. SETD1A methyltransferase activity depends on interactions with other proteins in a highly conserved complex, called 'complex of proteins associated with Set1' (COMPASS). COMPASS was originally defined in yeast ${ }^{6}$, and subsequently shown to be conserved in flies and mammals ${ }^{7-9}$.

SETD1A appears to be important for multiple aspects of cell cycle regulation. For example, SETD1mediated H3K4me3 activates $\beta$-catenin expression, which is required for proliferation of neuronal progenitor cells ${ }^{10}$. Furthermore, SETD1A has been shown to promote cell cycle progression by activating the expression of micro RNAs that suppress the antiproliferative gene BTG2, which plays a role in the transition between the $\mathrm{G} 1$ and $\mathrm{S}$ phase of the cell cycle ${ }^{11}$. Finally, H3K4 methylation by SETD1A is crucial in maintaining genome stability, especially during DNA replication, by protecting newly-replicated DNA from degradation ${ }^{12}$.

Heterozygous Loss-of-Function (LoF) variants of SETD1A have been identified in several human individuals from large schizophrenia cohort studies and have been linked to disruption of speech development and early-onset epilepsy ${ }^{13-15}$. Previously, LoF variants were also identified in a limited number of children with developmental delay ${ }^{14}$, suggesting that SETD1A variants are a common biological underpinning of the different aforementioned phenotypes. To provide further insight into the somatic and behavioral profiles, we characterised the neurodevelopmental syndrome associated 
with SETD1A heterozygous LoF variants in this study. This is a prerequisite to develop future guidelines and management directed towards the vulnerability to schizophrenia or other psychiatric diseases. In addition, to further establish the role of SETD1A in underlying biological processes, we studied the effect of SETD1A variants on genome stability in patient cell lines, and investigated the role of the Drosophila melanogaster orthologue, Set1, in postmitotic memory forming neurons of the fly brain.

\section{Methods}

\section{Patients}

We collected the molecular and clinical features of 15 unpublished individuals with de novo SETD1A variants by a collaboration facilitated by GeneMatcher ${ }^{16}$ in which several clinical groups independently identified individuals with developmental delay/intellectual disability (DD/ID) and related phenotypes with rare variants in SETD1A during routine diagnostic genetic testing. Clinical analysis of these patients was performed during regular consultations focusing on medical history, physical examination, and observational analysis of behavioral features. In all patients, exome sequencing and variant filtering were performed, according to the routine procedures at each institute $^{17-22}$.

We compared these data to available information from previously published cases with pathogenic SETD1A variants associated with schizophrenia and NDDs ${ }^{14,23,24}$. Ten individuals reported in these previous studies originate from cohorts diagnosed with schizophrenia (UK10K-Finns and Swedish cohorts) and six individuals originate from cohorts of individuals seen with NDDs (Deciphering Developmental Disorders (DDD) project, Northern Finland Intellectual Disability (NFID) study and Northern Finland Birth Cohorts (NFBC)). Clinical information of the previously reported individuals was only scarcely provided. 
To further assess the impact of these variants on cellular SETD1A functions, we first isolated and generated lymphoblastoid cell lines (LCL) from 3 patients (6, 13 and 15), and from two unrelated controls.

For further details on the methods used for cell culture, immunoblotting, antibodies and RNA isolation see Supplementary Data 1 - Methods.

\section{DNA Fibres}

DNA fibre analysis was carried out as described previously ${ }^{12}$. Briefly, cells were pulse-labelled with CldU and IdU (Sigma-Aldrich) for 20 min each before a $5 \mathrm{~h}$ exposure to $4 \mathrm{mM} \mathrm{HU}$, and at least 200 replication forks were analysed per experimental condition. Images were taken using a Nikon Eclipse Ni microscope equipped with a 60X oil lens, and were acquired and analysed using Elements v4.5 software (Nikon). The lengths of labelled tracts were measured using Image $\mathrm{J}^{25}$ and arbitrary length values were converted into micrometers using the scale bars created by the microscope.

\section{Drosophila Stocks and Genetics}

To study the role of Set1 in postmitotic memory forming neurons of the fly brain, flies were reared on a standard medium (cornmeal-sugar-yeast-agar) at $25^{\circ} \mathrm{C}$ and $70 \%$ humidity with a $12 \mathrm{~h}: 12 \mathrm{~h}$ light/dark cycle. R14H06-Gal4 flies express Gal4 under the control of a mushroom body (MB) specific enhancer from the rutabaga gene ${ }^{26,27} \cdot m$ Cherry $^{R N A i}, \operatorname{Set}^{\text {RNAi1 }}, \operatorname{Set}^{\text {RNAi2 }}$, and $\operatorname{Set}^{\text {RNAi3 }}$ were all generated by the Transgenic RNAi Project at Harvard University Medical School ${ }^{28}$ using a common donor strain, therefore these RNAi lines all share a common genetic background. $m C h e r r y{ }^{R N A i}$ was used as a control in these experiments because it shares a common genetic background with all of the tested RNAi lines, and represents any non-specific effects of a non-targeting RNAi. mCherry ${ }^{\text {RNAi }}$, Set $1^{\text {RNAi1 }}$, Set ${ }^{\text {RNAi2 }}$, and Set $1^{\text {RNAi3 }}$ were crossed to $R 14$ HO6-Gal4 for courtship conditioning and activity experiments, actin-Gal4 for lethality assays, and UAS-mCD8::GFP; R14H06-Gal4 for analysis of MB morphology. For more details on the acquisition of the used fly stocks, see Supplementary Data 1 Methods. 


\section{Courtship Conditioning}

Flies were trained and tested for short-term (STM) and long-term memory (LTM) 4 days after eclosion using the courtship conditioning assay as previously described ${ }^{29-31}$. For each fly pair a courtship index $(\mathrm{Cl})$ was calculated, which is the proportion of time spent courting over 10 minutes. The learning Index (LI) represents the percentage of reduction in courtship behavior due to training and is used to directly compare memory ability between different genotypes. $\mathrm{LI}$ is a single value that is calculated from the Cls using the formula: $\mathrm{LI}=\left(\right.$ mean $-\mathrm{Cl}_{\text {naive }}-$ mean- $\left.-\mathrm{Cl}_{\text {trained }}\right) /$ mean- $\mathrm{Cl}_{\text {naive }}$. For more details on the courtship conditioning procedures, see Supplementary Data 1 - Methods.

\section{Analysis of Activity and Sleep}

One- to five-day old flies were assayed for locomotor activity in $12 \mathrm{~h}: 12 \mathrm{~h}$ light/dark regimens after at least three days entrainment, for four full days using DAM5M monitors (Trikinetics, Waltham, MA). Locomotor data was collected in 1 minute bins, and a 5 minute period of inactivity was used to define sleep ${ }^{32,33}$. Sleep parameters were analysed using pySolo and plotted ${ }^{34}$. Dead animals were excluded from analysis by a combination of automated filtering and visual inspection of locomotor traces.

\section{Analysis of Mushroom Body Morphology}

Brains from one- to five-day old day old adult male flies expressing the membrane bound UASmCD8::GFP under the control of R14H06-Gal4 were dissected in 1x PBS. Dissected brains were fixed in 4\% paraformaldehyde for 50 minutes and then washed in a PBS with $1 \%$ Triton X-100 for 5 minutes. Brains were then mounted using Vectashield and imaged with a Zeiss LSM 710 confocal microscope. Confocal projections were created using ImageJ software (v. 2.0.0) ${ }^{25}$.

\section{Results}

\section{Patients: Molecular Phenotype}

An extensive overview of the clinical and molecular data of the patients included in this study is provided per individual in Supplementary Table 1. For one variant paternal genotype data was unavailable, therefore paternal inheritance could not be fully excluded. All other variants of the 
SETD1A gene identified in our cohort had occurred de novo. The 15 SETD1A variants identified here include 5 nonsense, 6 frameshifts, 1 missense and 2 different splice site variants, of which one occurred in two unrelated individuals. These 14 different identified SETD1A variants were all located before the conserved SET domain, which is responsible for catalyzing H3K4 methylation (Figure 1).

To gain further insight into the pathogenicity of the missense variant (Tyr1499Asp), we utilized several in silico prediction models. MetaDome is an online data platform that uses homologous domain relations and combines data from GnomAD with ClinVar to produce a 'tolerance landscape' of a gene ${ }^{35,36}$. According to MetaDome the region in which the variant lies is intolerant (Supplementary Figure 1). In addition, several other in silico prediction tools indicated that this variant is pathogenic: CADD PHRED: $29.7^{37}$; SIFT: Deleterious, score $0^{38}$; Polyphen-2: Probably damaging, score $1,000^{39}$.

In each patient, the observed SETD1A variant was considered to be the most likely contributor to the phenotype. In general, SETD1A is depleted from truncating variants in healthy individuals ( $p L i=1$ ).

Only 2 of the 14 different variants were listed in GnomAD: c.2968C>T and c.4582-2_4582delAG (with a low allele frequency of $4.061 \mathrm{e}^{-6}$ and $8.237 \mathrm{e}^{-6}$, respectively $)^{40}$. Interestingly, this latter variant, (c.4582-2_4582delAG) was identified in two different patients but had also been identified previously ${ }^{14}$. To determine any effects of this splice-site variant on the SETD1A transcript, RT-PCR was performed on cells from individual 15, amplifying a region between exon 14 and 18 . The resulting product shows that the transcript produced in individual 15 differs from the wildtype transcript. Sequencing of the amplified PCR-products revealed that the variant causes an insertion of 81 base pairs between exon 15 and 16, showing that intron 15 is not spliced. This insertion is in frame, but the N-SET domain, which is important for methyltransferase activity, is interrupted (Supplementary Figure 2). Preventing nonsense mediated RNA decay with cyclohexamide resulted in a 50:50 ratio of wildtype (WT) to mutant transcript. Without cyclohexamide the WT product was higher than mutant, 
suggesting that the mutant mRNA is partly degraded in the cell. These results suggest that this recurrent splice acceptor variant causes LoF by disrupting the N-SET domain of SETD1A.

\section{The Cellular Impact of SETD1A Variants}

Immunoblotting analyses revealed that cells from two patients, harboring the p.Gly535Alafs* and c.4582-2_4582delAG variants, exhibited decreased levels of SETD1A protein. The presence of the Tyr1499Asp variant had no effect on SETD1A protein levels (Figure 2A). Moreover, when these patient-derived cells were exposed to DNA damage in the form of high-dose hydroxyurea, they exhibited slightly elevated activation of the DNA damage response compared to WT counterparts, evidenced by increases in phosphorylation of CHK1 and RPA (Figure 2B).

More strikingly, SETD1A variants were also associated with severe levels of nascent DNA degradation upon DNA damage, to levels comparable with depletion of SETD1A ${ }^{12}$. This was not evident in control cells, but was apparent in all 3 patient-derived cell lines examined (Figure $2 \mathrm{C}$ ). These data suggest that these variants, including the Tyr1499Asp variant within the catalytic SET domain, may all lead to loss of SETD1A function. Moreover, given the severity of this defect, heterozygous variants in SETD1A mimic phenotypes that were previously observed in cell lines treated with SETD1A siRNA.

\section{Patients: Clinical Phenotype}

Our cohort of 15 individuals with heterozygous SETD1A variants comprised 8 females and 7 males whose ages varied from 34 months to 23 years (Supplementary Figure 3). The collection of clinical data of these individuals has allowed us to further define the clinical phenotype associated with SETD1A haploinsufficiency. A summary of the clinical data of the 15 patients included in this study is presented in Table 1. Figure 3 depicts the facial appearance of the patients for which portrait photographs were available. An extensive overview of the clinical features of the 15 individuals can be found in Supplementary Table 1. For comparison, we provide a synopsis of the clinical descriptions of the previously published cohort of 16 individuals with SETD1A variants ${ }^{14}$ in addition to 
our clinical data (Column 17 in Supplementary Table 1). For additional detailed clinical information per individual, see Supplementary Data 2 - Results.

\section{Development}

Global developmental delay was reported in 14/15 individuals, including delayed speech and language development (14/14) and motor development (13/14). In 6 patients formal IQ evaluations have been performed, confirming intellectual disability $(\mathrm{TIQ}<70)$ in $5 / 6$ patients. In the other individuals, observations in educational environments indicated learning difficulties or developmental delay in 6/9 individuals. The level of ID or developmental delay is generally mild.

\section{Behavioral and Psychiatric Abnormalities}

Because of the previously reported association between schizophrenia and variants in the SETD1A gene $^{14}$, we specifically looked at psychiatric and behavioral abnormalities in our patient cohort. Behavioral abnormalities were observed in 14/15 individuals. These consisted of aggressive behavior (5/14), anxiety (4/14), short attention span (4/14), autistic features (3/14), overfriendliness (3/14) and abnormal temper tantrums (2/14). In our cohort, 7 out of 12 patients suffered from sleep disturbances, including night terrors.

There were signs (though no formal diagnoses) of a psychotic disorder in two individuals in the cohort. First, case 15, a 23-year-old man with the recurrent splice acceptor change (c.45822_4582delAG), experienced periods with apparent negative symptoms associated with schizophrenia (somberness and social withdrawal), alternating with periods of euphoria. He was treated with aripiprazole, $10 \mathrm{mg}$ daily. He also showed aggressive and addictive behavior from a young age and has been diagnosed with PDD-NOS (DSM-IV criteria), which is part of the autism spectrum according to current diagnostic criteria (DSM-V) $)^{1}$. Second, case 3, a 16-year-old female, showed symptoms that could be classified as visual hallucinations and imperative auditory hallucinations, as she was reported to "hear voices telling her to do things and see things that are not there". This seemed to be transient and has not reoccurred. Because she was assessed as doing well, she so far has received neither formal evaluation nor medication for these symptoms. 


\section{Neurological Abnormalities}

Seizures were present in 3/15 individuals, with some very severe forms. For example, case 2 had myoclonic-astatic epilepsy. Drug treatment was sufficient to control seizures in case 2 and 3 , but for case 11 hemispherectomy was required for seizure control. Hypotonia was reported in 9/15 individuals, often manifesting during infancy and remained relatively mild. In 4/12 patients for which MRI has been performed, morphological abnormalities of the brain were reported including corpus callosum abnormalities, cerebral white matter abnormalities, T2 hyperintensities, and Chiari I malformation. Other reported neurological abnormalities are EEG abnormalities, encephalopathy, dysarthria, slight tremor, sensory processing disorder, and short-term memory deficit.

\section{Musculoskeletal Abnormalities}

Musculoskeletal abnormalities mainly affect the extremities (12/15) and included pes planus (6/12), broad/wide fingers (3/12), long fingers (2/12), and tapered fingers (2/12). Joint hypermobility was reported for $7 / 13$ individuals. Other, less penetrant musculoskeletal abnormalities included plagiocephaly (2/15), hyperlordosis (2/15), craniosynostosis (2/15), kyphosis (1/15), pectus excavatum (1/15), hip dysplasia (1/15), postural instability (1/15), right sided preference $(1 / 15)$, and congenital torticollis (1/15).

\section{Gastrointestinal Abnormalities}

Gastrointestinal abnormalities were present in $8 / 12$ individuals. Feeding difficulties were reported for $3 / 8$ individuals. The feeding difficulties mainly occurred and resolved during infancy. Constipation was observed in $3 / 8$ individuals. Less common features included gastroesophageal reflux, frequent bowel movements and insatiable appetite.

For additional clinical information on highlighted individual cases see Supplementary Data 2 - Results.

\section{Drosophila: Memory Phenotype}

To gain insight into the function of SETD1A in neurons, we investigated Drosophila Set1, which is an orthologue of human SETD1A and SETD1B. Both SETD1A and SETD1B are mutated in individuals with overlapping neurodevelopmental disorders ${ }^{41}$. The Set1 family of proteins are known to be important 
regulators of cell proliferation and differentiation ${ }^{10-12,42,43}$. However, phenotypes that are normally associated with altered cell proliferation, i.e. overgrowth and cancer, are not frequently observed in SETD1A or SETD1B patient cohorts. To gain insight into the role of Drosophila Set1 in other aspects of neurobiology, we investigated terminally differentiated postmitotic neurons of the Drosophila mushroom body $(\mathrm{MB})$, the primary learning and memory center of the fly brain.

Since germline Set1 mutants are lethal ${ }^{44}$, we used the spatially restricted Gal4/UAS system ${ }^{45}$ to induce targeted RNA interference (RNAi) of Set1. Postmitotic MB neurons were targeted using the R14H06-Gal4 driver line, which we have characterised previously ${ }^{26,27}$. $R 14$ H06-Gal4 is highly specific for the MB in adult and larval fly brains ${ }^{46}$. It is also expressed in a few cells of the ventral nerve chord, which appear to be chordotonal sensory neurons involved in balance and locomotion. We obtained three different Set1 UAS-RNAi transgenes that target different parts of the Set1 mRNA. We tested the RNAi potency by crossing RNAi stocks to the ubiquitous actin-Gal4 driver. As Set1 is an essential gene this would be expected to induce lethality. While $\operatorname{Set} 1^{\text {RNAi1 }}$ and $\operatorname{Set} 1^{\text {RNAi2 }}$ did induce lethality, $\operatorname{Set} 1^{\text {RNAi3 }}$ did not, suggesting that the $\operatorname{Set} 1^{\text {RNAi3 }}$ line is less potent. As a control, we used an mCherry RNAi strain (mCherry ${ }^{R N A i}$ ) that had the same genetic background as the Set1 RNAi strains.

We tested the effect of postmitotic Set1 knockdown on memory using the courtship conditioning paradigm, a classic memory assay based on naturally occurring courtship behaviors ${ }^{29}$. Socially naïve male flies court female flies vigorously, however previously mated females reject male courtship attempts. In the presence of normal learning and memory, male flies reduce futile courtship behavior in response to sexual rejection by a non-receptive mated female. In this assay, male flies are trained by pairing with a premated female that rejects male courtship attempts. After 1-hour of training, the $\mathrm{Cl}$ was significantly reduced in the mCherry ${ }^{\mathrm{RNAi}}$ control and the less potent Set $1^{\text {RNAi3 }}$ when compared to naïve flies of the same genotype, indicating normal short-term memory (STM) (Figure 4A, left panel). In contrast, there was no significant reduction in $\mathrm{Cl}$ after training for Set $1^{\text {RNAi1 }}$ and Set $1^{\text {RNAi2 }}$, indicating defective memory upon Set1 knockdown in postmitotic MB neurons. We observed similar 
results for long-term memory (LTM) (Figure 4A, right panel) with the mCherry ${ }^{\text {RNAi }}$ and Set $1^{\text {RNAi3 }}$ flies showing a significant reduction in $\mathrm{Cl}$ in response to sexual rejection, and no significant reduction for Set $1^{\text {RNAi1 }}$ and Set $1^{\text {RNAi2 }}$. Set $1^{\text {RNAi1 }}$ and Set $1^{\text {RNAi2 }}$ showed a significantly reduced $\mathrm{LI}$ when compared to the control for both STM and LTM, while Set1 ${ }^{\text {RNAi3 }}$ did not (Figure 4B). Taken together, these data suggest that Set1 is required in postmitotic MB neurons for normal memory.

We also assessed sleep, activity, and baseline courtship behavior in MB-specific Set1 knockdown flies. There was a small but significant $(p=0.03)$ reduction in sleep for Set $1^{\text {RNAi1 }}$, however, this reduction was not observed for Set1 $1^{\text {RNAi2 }}$ (Supplementary Figure 4A). Overall activity (Supplementary Figure 4B) and naïve courtship levels (Supplementary Figure 4C) were not significantly different in Set $1^{\text {RNAi1 }}$ and Set $1^{\text {RNAi2 }}$ when compared to the control. This suggests that courtship conditioning defects are not due to defects in locomotion or baseline courtship ability. It also suggests that memory defects likely arise from the role of Set 1 in the MB cells, and not the chordotonal sensory neurons that are also targeted by R14H06-Gal4. Disruption of chordotonal organ function would be expected to cause severe defects in balance and locomotion, which clearly are not observed upon Set1 knockdown with R14H06-Gal4.

R14H06-Gal4 is expressed in the larval MB as well as the adult MB. During larval and pupal development, postmitotic MB neurons undergo morphological changes that are required for normal wiring of the adult fly brain. We asked if memory defects in MB Set1 knockdown flies result from morphological abnormalities arising from defects in MB development. To test this, we labelled R14H06-Gal4 neurons by co-expression of a membrane targeted green fluorescent protein (GFP) and imaged whole mount adult brains using confocal microscopy. We did not observe gross morphological defects in larval, pupal, or adult stages in Set1 knockdown flies (Supplementary Figure 4D). Thus, Set1 appears to be dispensable in postmitotic neurons for normal MB morphogenesis. Taken together with our behavior data, this suggests that Set1 is required for normal functioning of adult MB neurons during the formation of short- and long-term courtship memory. 


\section{Discussion}

Characterization of a Novel Neurodevelopmental Disorder caused by SETD1A Haploinsufficiency

The presented clinical and molecular data strongly support the conclusion that heterozygous LoF of human SETD1A causes a novel defined neurodevelopmental disorder. The core features associated with SETD1A haploinsufficiency include global developmental delay or intellectual disability, subtle facial dysmorphisms, and psychiatric problems. In previous studies heterozygous LoF variants in SETD1A were observed in individuals with schizophrenia ${ }^{14}$. Therefore, in this study we investigated the behavioral and psychiatric abnormalities that have been observed in our predominantly pediatric cohort. No patient had a previous diagnosis of schizophrenia, but this is expected because of the relatively young age of the patients. With the exception of the very rare childhood onset forms ${ }^{47}$, schizophrenia rarely manifests so early ${ }^{48}$. Interestingly, an adolescent and a young adult from our cohort (age 16 years and 23 years) showed signs that could be linked with schizophrenia or bipolar disorder, though they had no formal psychiatric diagnosis. Combined with the previous reports, this indicates that the germline SETD1A variants might predispose individuals to severe neuropsychiatric pathologies later in life. Therefore, this patient group should be carefully monitored for suggestive psychiatric symptoms.

It is notable that in humans all six H3K4 methyltransferases, SETD1A/B, KMT2A/B, and $K M T 2 C / D$ have been implicated in neurodevelopmental disorders, though it is also clear that all KMT2 members have distinct molecular functions ${ }^{30,41,49-51}$. However, there is an interesting overlap between these chromatin modulating genes and their respective phenotypes (with developmental delay/intellectual disability at its core), based on their shared underlying disease mechanisms $\mathrm{s}^{52}$.

\section{SETD1A Mutational Mechanisms and Cellular Phenotypes}

In the 15 patients investigated in this study we identified 14 different SETD1A variants. The majority of these were LoF nonsense or frameshift variants, however a twice recurrent splice-site variant and a missense variant were also identified in the catalytic SET domain, which is required for 
methyltransferase activity. Previously, SETD1A-mediated H3K4 methylation was shown to be important for protecting against DNA damage at stalled replication forks ${ }^{12}$. We used this cellular phenotype as an output to test the functional effects of clinical SETD1A variants in three patient derived lymphoblastoid cell lines. Interestingly, cell lines with heterozygous LoF alleles (p.Gly535Alafs*12, and c.4582-2_4582delAG) showed high levels of nascent DNA degradation, at a level of severity that was similar to that observed in cell lines treated with SETD1A siRNA ${ }^{12}$. Moreover, cells with the heterozygous missense variant (p.Tyr1499Asp) also showed a similar phenotype, thus supporting the pathogenicity of this variant. Taken together, these results suggest that SETD1A-associated clinical features likely result from partial loss of SETD1A methyltransferase activity.

In two unrelated patients we found the same de novo two base pair (bp) deletion (c.4582-2_45821delAG) at the splice acceptor of exon 16. The Human Splicing Finder (version 3.1) predicts that this two bp alteration of the acceptor splice site of exon 16 results in exon skipping ${ }^{53}$. Interestingly, in non-pathogenic situations the overall expression of exon 16 is the highest compared to the other exons of the gene in different tissues ${ }^{54}$ (Supplementary Figure 5 ). This suggests a significant role for this particular exon for the functionality of the N-SET domain, which is partly encoded by this exon. Our RNA analyses surprisingly revealed that exon skipping did not occur, but the two bp deletion rather resulted in retention of intron 15. In the three other studies describing patients with variants in SETD1A, the exact same variant was found in 7 out of the total of 16 patients ${ }^{14,23,24}$. In addition, this variant occurs at a frequency of $8.071 \mathrm{e}^{-6}$ in the GnomAD database ${ }^{40}$. Authors from the previous studies did not speculate on this seemingly unlikely recurrence of the two bp deletion ${ }^{14,24}$. However, it raises questions on the frequency and etiology of occurrence of this variant. It is possible that the mutation rate in this genomic area, or specifically around these two base pairs, is relatively high. This may be due to the surrounding sequence homology or the epigenetic state of the DNA, which in turn may allow this deletion to occur. Another explanation might be ascertainment bias, due to the possibility that the specific variant gives rise to ID and/or schizophrenia. Whereas other SETD1A 
variants might also be causative for other phenotypes and therefore would not be included in studies focusing on ID or schizophrenia. In addition, it might be that the c.4582-2_4582-1delAG variant is a so-called selfish mutation. Certain DNA variants that can occur over time in spermatogonial cells can lead to clonal expansion (such as the c.755C>G variant in the FGFR2 gene in the case of Apert syndrome). This can culminate to the passing of that certain variant from father to child, leading to a higher prevalence in the patient group. Typically these variants are 'Gain of Function' $(\mathrm{GoF})^{55,56}$, so in our cohort this does not seem to be the case.

\section{The Drosophila SETD1A Orthologue is required in Postmitotic Neurons for Memory}

SETD1A and other COMPASS components have been implicated in the regulation of cell proliferation and differentiation. However, there is little evidence that these processes solely underlie the clinical features that we describe in SETD1A haploinsufficiency, as the cognitive deficits and facial dysmorphisms reported in our cohort are mild. Defects in proliferation or differentiation of neural progenitors might have more dramatic effects on structure and function of the brain, and cause profound cognitive deficits or lethality. In line with this, we identified a role for Drosophila Set1 in the regulation of memory formation in postmitotic neurons. This finding is consistent with studies demonstrating a role for different histone modifications in the epigenetic regulation of memory ${ }^{57}$. For example, $\mathrm{H} 3 \mathrm{~K} 4 \mathrm{me} 3$ is dynamically regulated in the rodent hippocampus during memory formation and Setd1 $\mathrm{a}^{+/-}$mice exhibit deficits in working memory ${ }^{58,59}$. Several other H3K4 methyltransferases have also been implicated in memory, including mouse Kmt2a/Kmt2b and Drosophila $\operatorname{trr}^{30,49,60}$. Thus, our findings on SETD1A and Drosophila Set1 support a growing body of literature highlighting the conserved role of H3K4 methylation in regulating the function of adult neurons post development.

The specific role of H3K4 methylation in memory formation is not known. Set1-related proteins might regulate several different aspects of postmitotic neuronal biology that could all impact memory. For example, we have shown here that patient cell lines have elevated damage in nascent 
DNA. Terminally differentiated neurons are often exposed to DNA damage and an efficient response is critical, as these neurons are not easily replaced. Additionally, the Set1 protein family might be important for maintaining the cell-type specific gene expression programs that would be critical for normal functioning of memory neurons. Set1-related proteins might also be required for induction of learning-induced gene expression changes that are known to be required for long-term memory formation.

Despite the lack of a precise pathogenetic mechanism, the finding that Set1 regulates memory post development could have implications for therapies. Further understanding of SETD1A function in the postnatal brain could open up possibilities for therapy that would not be possible if pathogenic mechanisms occurred entirely during the prenatal stages of development.

\section{Compliance with ethical standards}

All procedures performed in studies involving human participants were in accordance with the ethical standards of the institutional and/or national research committee and with the 1964 Helsinki declaration and its later amendments or comparable ethical standards.

Informed consent was obtained from all individual participants included in the study as part of the diagnostic workflow. Additional informed consent was obtained from all individual participants for whom identifying information is included in this article.

\section{Acknowledgements}

We are grateful to all individuals and their parents for participating in this study.

This work was supported by the Netherlands Organization for Health Research and Development (ZonMw grant 91718310) to T.K., ERA-NET NEURON-102 SYNSCHIZ (grant 013-17-003 4538) to D.S., Canadian Institutes of Health Research, Canadian Foundation for Innovation, and the Canada Research Chairs Program to J.M.K., MRC Career Development Fellowship (MR/P009085/1) and a University of Birmingham Fellowship to M.R.H. 
We thank the Bloomington Drosophila Stock Center at Indiana University for providing all Drosophila strains used in this study.

Sequencing and analysis for patient 2 were provided by the Broad Institute of MIT and Harvard Center for Mendelian Genomics (Broad CMG) and was funded by the National Human Genome Research Institute, the National Eye Institute, and the National Heart, Lung and Blood Institute grant UM1 HG008900 and in part by National Human Genome Research Institute grant R01 HG009141.

\section{Conflict of interest}

All contributors have read and approved the submission to the journal.

M.T. Cho and K. McWalter are employees of GeneDx. We are not aware of any other conflict of interest.

Supplementary information is available at MP's website.

\section{References}

1. Association AP. Diagnostic and statistical manual of mental disorders. 5th edn. American Psychiatric Publishing: Arlington, VA, 2013.

2. Beckwith EJ, Geissmann Q, French AS, Gilestro GF. Regulation of sleep homeostasis by sexual arousal. eLife 2017; 6.

3. De Rubeis S, He X, Goldberg AP, Poultney CS, Samocha K, Cicek AE et al. Synaptic, transcriptional and chromatin genes disrupted in autism. Nature 2014; 515(7526): 209-215.

4. Iossifov I, O'Roak BJ, Sanders SJ, Ronemus M, Krumm N, Levy D et al. The contribution of de novo coding mutations to autism spectrum disorder. Nature 2014; 515(7526): 216-221.

5. Lee JH, Skalnik DG. Wdr82 is a C-terminal domain-binding protein that recruits the Setd1A Histone H3-Lys4 methyltransferase complex to transcription start sites of transcribed human genes. Molecular and cellular biology 2008; 28(2): 609-618.

6. Miller T, Krogan NJ, Dover J, Erdjument-Bromage $H$, Tempst $\mathrm{P}$, Johnston $\mathrm{M}$ et al. COMPASS: a complex of proteins associated with a trithorax-related SET domain protein. Proceedings of the National Academy of Sciences of the United States of America 2001; 98(23): 12902-

12907. 
7. Mohan M, Herz HM, Smith ER, Zhang Y, Jackson J, Washburn MP et al. The COMPASS family of H3K4 methylases in Drosophila. Molecular and cellular biology 2011; 31(21): 4310-4318.

8. Takahashi YH, Westfield GH, Oleskie AN, Trievel RC, Shilatifard A, Skiniotis G. Structural analysis of the core COMPASS family of histone H3K4 methylases from yeast to human. Proceedings of the National Academy of Sciences of the United States of America 2011; 108(51): 20526-20531.

9. Shilatifard A. The COMPASS family of histone H3K4 methylases: mechanisms of regulation in development and disease pathogenesis. Annual review of biochemistry 2012; 81: 65-95.

10. Li Y, Jiao J. Histone chaperone HIRA regulates neural progenitor cell proliferation and neurogenesis via beta-catenin. The Journal of cell biology 2017; 216(7): 1975-1992.

11. Tajima K, Yae T, Javaid S, Tam O, Comaills V, Morris R et al. SETD1A modulates cell cycle progression through a miRNA network that regulates p53 target genes. Nat Commun 2015; 6: 8257.

12. Higgs MR, Sato K, Reynolds JJ, Begum S, Bayley R, Goula A et al. Histone Methylation by SETD1A Protects Nascent DNA through the Nucleosome Chaperone Activity of FANCD2. Molecular cell 2018; 71(1): 25-41.e26.

13. Eising E, Carrion-Castillo A, Vino A, Strand EA, Jakielski KJ, Scerri TS et al. A set of regulatory genes co-expressed in embryonic human brain is implicated in disrupted speech development. Molecular psychiatry 2018.

14. Singh T, Kurki MI, Curtis D, Purcell SM, Crooks L, McRae J et al. Rare loss-of-function variants in SETD1A are associated with schizophrenia and developmental disorders. Nat Neurosci 2016; 19(4): 571-577.

15. Yu X, Yang L, Li J, Li W, Li D, Wang R et al. De Novo and Inherited SETD1A Variants in Earlyonset Epilepsy. Neuroscience bulletin 2019.

16. Sobreira N, Schiettecatte F, Valle D, Hamosh A. GeneMatcher: a matching tool for connecting investigators with an interest in the same gene. Human mutation 2015; 36(10): 928-930.

17. Neveling K, Feenstra I, Gilissen C, Hoefsloot LH, Kamsteeg EJ, Mensenkamp AR et al. A posthoc comparison of the utility of sanger sequencing and exome sequencing for the diagnosis of heterogeneous diseases. Human mutation 2013; 34(12): 1721-1726.

18. Retterer K, Juusola J, Cho MT, Vitazka P, Millan F, Gibellini F et al. Clinical application of whole-exome sequencing across clinical indications. Genetics in medicine : official journal of the American College of Medical Genetics 2016; 18(7): 696-704. 
19. Boonsawat P, Joset P, Steindl K, Oneda B, Gogoll L, Azzarello-Burri S et al. Elucidation of the phenotypic spectrum and genetic landscape in primary and secondary microcephaly. Genetics in medicine : official journal of the American College of Medical Genetics 2019.

20. Lessel D, Gehbauer C, Bramswig NC, Schluth-Bolard C, Venkataramanappa S, van Gassen KLI et al. BCL11B mutations in patients affected by a neurodevelopmental disorder with reduced type 2 innate lymphoid cells. Brain : a journal of neurology 2018; 141(8): 2299-2311.

21. Gudbjartsson DF, Helgason H, Gudjonsson SA, Zink F, Oddson A, Gylfason A et al. Large-scale whole-genome sequencing of the Icelandic population. Nature genetics 2015; 47(5): 435-444.

22. Weiss K, Terhal PA, Cohen L, Bruccoleri M, Irving M, Martinez AF et al. De Novo Mutations in CHD4, an ATP-Dependent Chromatin Remodeler Gene, Cause an Intellectual Disability Syndrome with Distinctive Dysmorphisms. American journal of human genetics 2016; 99(4): 934-941.

23. Takata A, Xu B, lonita-Laza I, Roos JL, Gogos JA, Karayiorgou M. Loss-of-function variants in schizophrenia risk and SETD1A as a candidate susceptibility gene. Neuron 2014; 82(4): 773780.

24. Guipponi MS, FA; Setola, V; Gehrig, C; Rotharmel, M;. Exome Sequencing in 53 Sporadic Cases of Schizophrenia Identifies 18 Putative Candidate Genes 2014.

25. Schneider CA, Rasband WS, Eliceiri KW. NIH Image to ImageJ: 25 years of image analysis. Nat Methods 2012; 9(7): 671-675.

26. Jenett A, Rubin GM, Ngo TT, Shepherd D, Murphy C, Dionne H et al. A GAL4-driver line resource for Drosophila neurobiology. Cell reports 2012; 2(4): 991-1001.

27. Jones SG, Nixon KCJ, Chubak MC, Kramer JM. Mushroom Body Specific Transcriptome Analysis Reveals Dynamic Regulation of Learning and Memory Genes After Acquisition of Long-Term Courtship Memory in Drosophila. G3 (Bethesda, Md) 2018; 8(11): 3433-3446.

28. Perkins LA, Holderbaum L, Tao R, Hu Y, Sopko R, McCall K et al. The Transgenic RNAi Project at Harvard Medical School: Resources and Validation. Genetics 2015; 201(3): 843-852.

29. Siegel RW, Hall JC. Conditioned responses in courtship behavior of normal and mutant Drosophila. Proceedings of the National Academy of Sciences of the United States of America 1979; 76(7): 3430-3434. 
30. Koemans TS, Kleefstra T, Chubak MC, Stone MH, Reijnders MRF, de Munnik S et al. Functional convergence of histone methyltransferases EHMT1 and KMT2C involved in intellectual disability and autism spectrum disorder. PLoS genetics 2017; 13(10): e1006864.

31. Koemans TS, Oppitz C, Donders RAT, van Bokhoven H, Schenck A, Keleman K et al. Drosophila Courtship Conditioning As a Measure of Learning and Memory. Journal of visualized experiments : JoVE 2017; (124).

32. Shaw PJ, Cirelli C, Greenspan RJ, Tononi G. Correlates of sleep and waking in Drosophila melanogaster. Science (New York, NY) 2000; 287(5459): 1834-1837.

33. Huber R, Hill SL, Holladay C, Biesiadecki M, Tononi G, Cirelli C. Sleep homeostasis in Drosophila melanogaster. Sleep 2004; 27(4): 628-639.

34. Gilestro GF, Cirelli C. pySolo: a complete suite for sleep analysis in Drosophila. Bioinformatics (Oxford, England) 2009; 25(11): 1466-1467.

35. Bock I, Nemeth K, Pentelenyi K, Balicza P, Balazs A, Molnar MJ et al. Targeted next generation sequencing of a panel of autism-related genes identifies an EHMT1 mutation in a Kleefstra syndrome patient with autism and normal intellectual performance. Gene 2016; 595(2): 131141.

36. Wiel L, Venselaar H, Veltman JA, Vriend G, Gilissen C. Aggregation of population-based genetic variation over protein domain homologues and its potential use in genetic diagnostics. Human mutation 2017; 38(11): 1454-1463.

37. Rentzsch P, Witten D, Cooper GM, Shendure J, Kircher M. CADD: predicting the deleteriousness of variants throughout the human genome. Nucleic acids research 2019; 47(D1): D886-d894.

38. Sim NL, Kumar P, Hu J, Henikoff S, Schneider G, Ng PC. SIFT web server: predicting effects of amino acid substitutions on proteins. Nucleic acids research 2012; 40(Web Server issue): W452-457.

39. Adzhubei IA, Schmidt S, Peshkin L, Ramensky VE, Gerasimova A, Bork P et al. A method and server for predicting damaging missense mutations. Nature methods 2010; 7(4): 248-249.

40. Lek M, Karczewski KJ, Minikel EV, Samocha KE, Banks E, Fennell T et al. Analysis of proteincoding genetic variation in 60,706 humans. Nature 2016; 536(7616): 285-291.

41. Hiraide $\mathrm{T}$, Nakashima $\mathrm{M}$, Yamoto $\mathrm{K}$, Fukuda $\mathrm{T}$, Kato $\mathrm{M}$, Ikeda $\mathrm{H}$ et al. De novo variants in SETD1B are associated with intellectual disability, epilepsy and autism. Hum Genet 2018; 137(1): 95-104. 
42. Blobel GA, Kadauke S, Wang E, Lau AW, Zuber J, Chou MM et al. A reconfigured pattern of MLL occupancy within mitotic chromatin promotes rapid transcriptional reactivation following mitotic exit. Molecular cell 2009; 36(6): 970-983.

43. Petruk S, Sedkov Y, Johnston DM, Hodgson JW, Black KL, Kovermann SK et al. TrxG and PcG proteins but not methylated histones remain associated with DNA through replication. Cell 2012; 150(5): 922-933.

44. Hallson G, Hollebakken RE, Li T, Syrzycka M, Kim I, Cotsworth S et al. dSet1 is the main H3K4 di- and tri-methyltransferase throughout Drosophila development. Genetics 2012; 190(1): 91-100.

45. Brand AH, Manoukian AS, Perrimon N. Ectopic expression in Drosophila. Methods in cell biology 1994; 44: 635-654.

46. Chubak MC, Nixon KCJ, Stone MH, Raun N, Rice SL, Sarikahya M et al. Individual components of the SWI/SNF chromatin remodelling complex have distinct roles in memory neurons of the Drosophila mushroom body. Dis Model Mech 2019; 12(3).

47. Driver DI, Gogtay N, Rapoport JL. Childhood onset schizophrenia and early onset schizophrenia spectrum disorders. Child and adolescent psychiatric clinics of North America 2013; 22(4): 539-555.

48. Rajji TK, Ismail Z, Mulsant BH. Age at onset and cognition in schizophrenia: meta-analysis. Br J Psychiatry 2009; 195(4): 286-293.

49. Kerimoglu C, Sakib MS, Jain G, Benito E, Burkhardt S, Capece V et al. KMT2A and KMT2B Mediate Memory Function by Affecting Distinct Genomic Regions. Cell reports 2017; 20(3): 538-548.

50. Ng SB, Bigham AW, Buckingham KJ, Hannibal MC, McMillin MJ, Gildersleeve HI et al. Exome sequencing identifies MLL2 mutations as a cause of Kabuki syndrome. Nature genetics 2010; 42(9): 790-793.

51. Faundes V, Newman WG, Bernardini L, Canham N, Clayton-Smith J, Dallapiccola B et al. Histone Lysine Methylases and Demethylases in the Landscape of Human Developmental Disorders. American journal of human genetics 2018; 102(1): 175-187.

52. McCarthy SE, Gillis J, Kramer M, Lihm J, Yoon S, Berstein $\mathrm{Y}$ et al. De novo mutations in schizophrenia implicate chromatin remodeling and support a genetic overlap with autism and intellectual disability. Molecular psychiatry 2014; 19(6): 652-658. 
53. Desmet FO, Hamroun D, Lalande M, Collod-Beroud G, Claustres M, Beroud C. Human Splicing Finder: an online bioinformatics tool to predict splicing signals. Nucleic acids research 2009; 37(9): e67.

54. Varga E, Nemes C, Tancos Z, Bock I, Berzsenyi S, Levay G et al. Establishment of EHMT1 mutant induced pluripotent stem cell (iPSC) line from a 11-year-old Kleefstra syndrome (KS) patient with autism and normal intellectual performance. Stem cell research 2016; 17(3): 531-533.

55. Goriely A, McGrath JJ, Hultman CM, Wilkie AO, Malaspina D. "Selfish spermatogonial selection": a novel mechanism for the association between advanced paternal age and neurodevelopmental disorders. The American journal of psychiatry 2013; 170(6): 599-608.

56. Goriely A, Wilkie AO. Paternal age effect mutations and selfish spermatogonial selection: causes and consequences for human disease. American journal of human genetics 2012; 90(2): 175-200.

57. Kim S, Kaang BK. Epigenetic regulation and chromatin remodeling in learning and memory. Experimental \& molecular medicine 2017; 49(1): e281.

58. Mukai J, Cannavo E, Crabtree GW, Sun Z, Diamantopoulou A, Thakur P et al. Recapitulation and Reversal of Schizophrenia-Related Phenotypes in Setd1a-Deficient Mice. Neuron 2019.

59. Gupta S, Kim SY, Artis S, Molfese DL, Schumacher A, Sweatt JD et al. Histone methylation regulates memory formation. The Journal of neuroscience : the official journal of the Society for Neuroscience 2010; 30(10): 3589-3599.

60. Kerimoglu C, Agis-Balboa RC, Kranz A, Stilling R, Bahari-Javan S, Benito-Garagorri E et al. Histone-methyltransferase MLL2 (KMT2B) is required for memory formation in mice. The Journal of neuroscience : the official journal of the Society for Neuroscience 2013; 33(8): 3452-3464.

61. Hunter S, Apweiler R, Attwood TK, Bairoch A, Bateman A, Binns D et al. InterPro: the integrative protein signature database. Nucleic acids research 2009; 37(Database issue): D211-215. 
Figure legends

Figure 1

The genomic position and coding consequences of 14 different SETD1A variants. All variants are located 5 ' to the conserved SET domain, which is responsible for catalyzing methylation. The splice acceptor variant at exon 16 (c.4582-2delAG) was identified in two unrelated patients. Mapping of the different domains (IPR024657, IPR001214, IPR000504 and IPR003616) on the gene was done according to data retrieved from Interpro ${ }^{61}$.

Figure 2

(A) Whole cell extracts (WCE) from the indicated patient-derived lymphoblastoid cell lines (LCL) were analysed by immunoblotting using the denoted antibodies. Data is derived from the untreated samples in (B).

(B) Patient-derived LCL were exposed to $2 \mathrm{mM} \mathrm{HU}$ for $24 \mathrm{~h}$, WCE prepared and analysed by immunoblotting using the denoted antibodies. Data is representative of $n=2$ independent experiments. * Denotes a non-specific band.

(C) Patient-derived LCL were sequentially pulsed for $20 \mathrm{~min}$ each with CldU and IdU, and exposed to 4 $\mathrm{mM} \mathrm{HU}$ for $5 \mathrm{~h}$ (as in the schema). DNA was visualised with antibodies to CldU and IdU, and tract length was calculated. Plots denote the average ratios of IdU:CldU label length from three independent experiments. Red lines indicate mean ratios. Data represents pooled values from $n=3$ experiments, representative images are shown.

Figure 3

Clinical portrait photographs of patients with a SETD1A pathogenic variant. The most prominent facial features to be observed are high forehead (7/15), low-set ears (4/15), microtia (3/15), downslanted palpebral fissures (6/15), epicanthus (7/15), deeply set eyes (4/15), hypertelorism (4/15), wide nose (6/15), anteverted nares (4/15), full cheeks (2/15), everted/tented upper lip 
vermillion (8/15), wide mouth (4/15) and widely spaced teeth (3/15).

Figure 4

Mushroom body specific knockdown of Set1 causes defects in short- and long-term memory.

(A) Courtship memory was measured in control flies and in flies that express Set1 RNAi constructs localized to the mushroom body by the R14H06-Gal4 driver. Three Set1 RNAi lines were compared to the mCherry ${ }^{\mathrm{RNAi}}$ control, which is present in the same genetic background as the Set1 RNAi lines. Boxplots show the distribution of Courtship Indices (Cls) for naïve $(\mathrm{N})$ and trained $(\mathrm{T})$ flies of control and Set1 RNAi lines. $\left({ }^{*}\right)$ And $(* * * *)$ indicate a significant reduction in $\mathrm{Cl}$ trained flies compared to naïve (Mann-Whitney Test; $p<0.05$ and $p<0.0001$, respectively). + Indicates the mean. $\mathrm{N}$ is indicated along $\mathrm{x}$-axis.

(B) Bar graphs showing the Learning indices (LI), which are single values that are derived from the above $\mathrm{Cls}$ according to the formula: $\mathrm{LI}=\left(\right.$ mean $\mathrm{Cl}_{\text {naive }}-$ mean $\left.\mathrm{Cl}_{\text {trained }}\right) /$ mean $\left.\mathrm{Cl}_{\text {naive }}\right) . \mathrm{LI}$ was significantly reduced for Set1 $^{\mathrm{RNAi1}}$ and Set1 ${ }^{\mathrm{RNAi2}}$, but not Set1 $1^{\mathrm{RNAi} 3} \cdot{ }^{* *} \mathrm{p}<0.01, * * * * p<0.0001$, randomization test, 10,000 bootstrap replicates. Genotypes are indicated below the $x$-axis.

Table legends

Table 1

Summary of clinical features found in the cohort of 15 individuals with SETD1A variants. *

* More extensive clinical information per individual is provided in Supplementary Table 1. As information on the different features was not always applicable or known for each patient, the denominator in the 'Frequency' column is different for different clinical characteristics. 


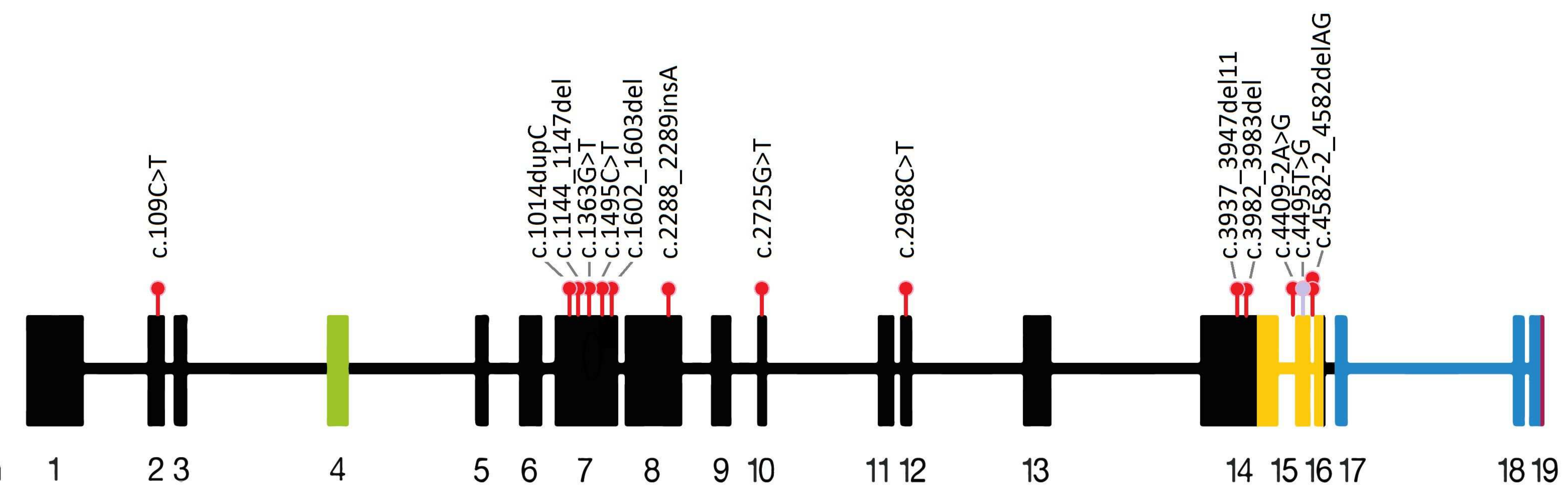

i Mutation

Missense mutation

- RNA recognition $\quad$ COMPASS complex Set1 motif (RRM) subunit, N-SET domain
- SET Domain
- Post-SET Domain 
A
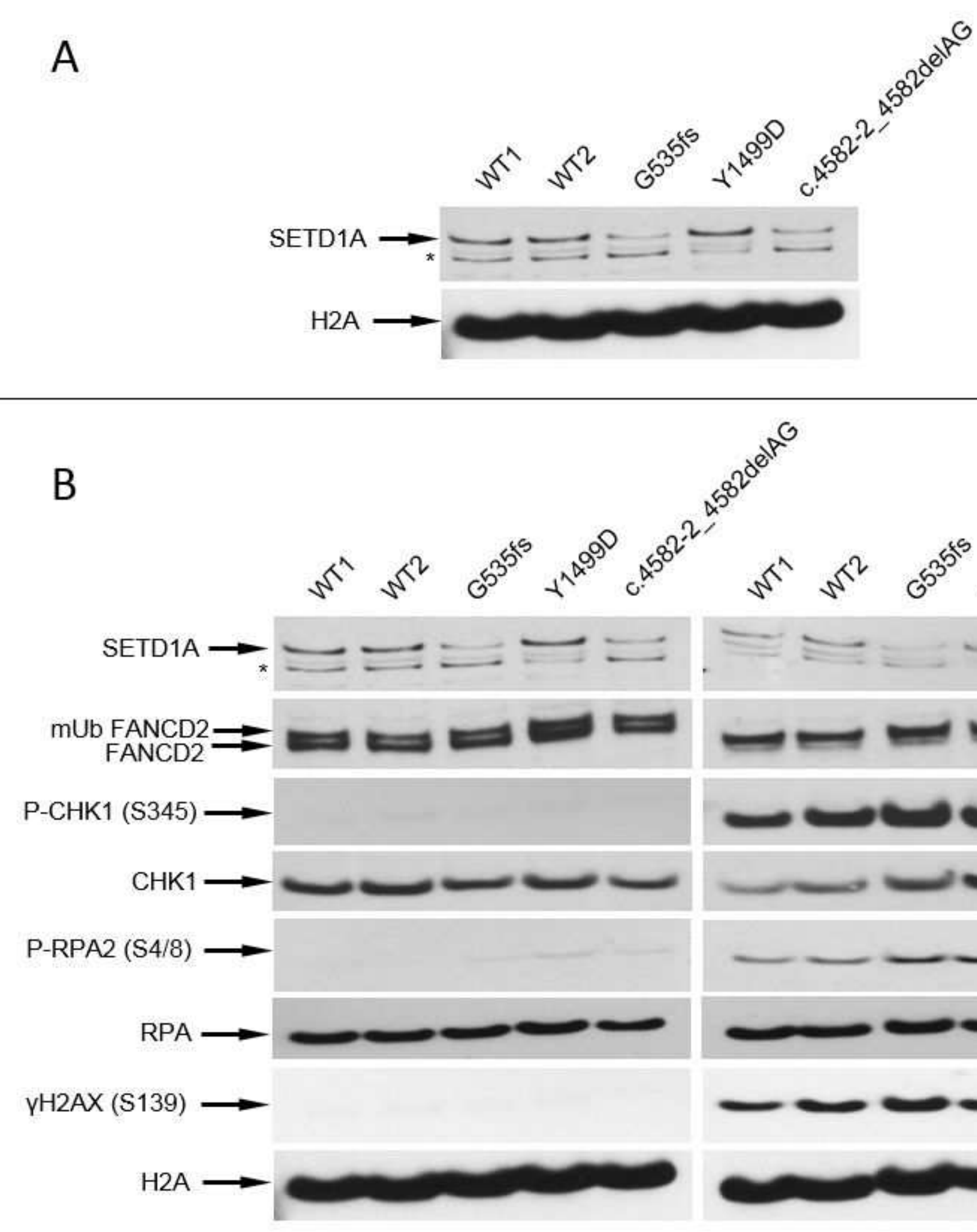

C

$\frac{\mathrm{CldU}}{20^{\prime}} \frac{\mathrm{IdU}}{20^{\prime}} \frac{\mathrm{HU}}{4 \mathrm{mM} ; 5 \mathrm{~h}}$
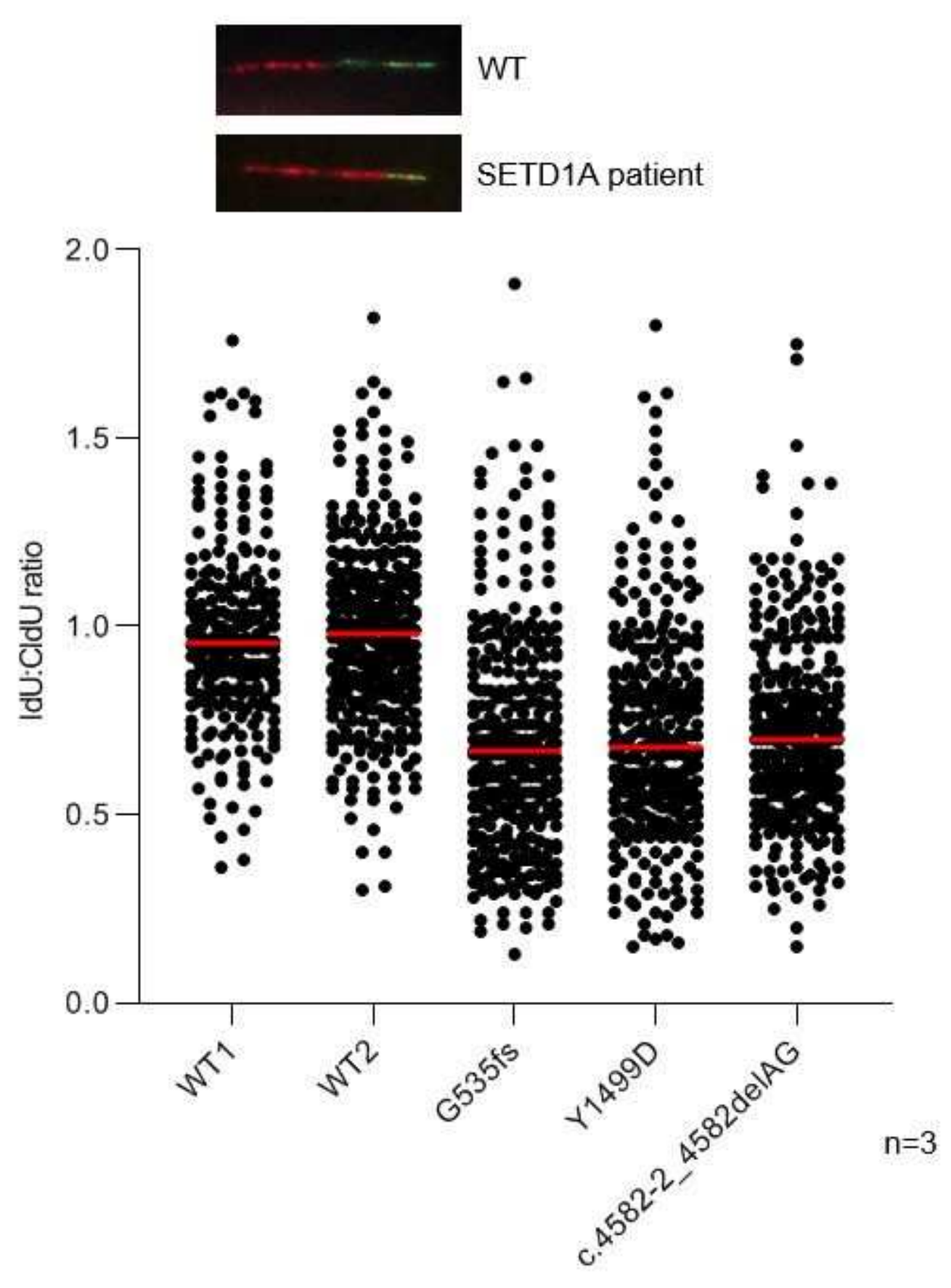


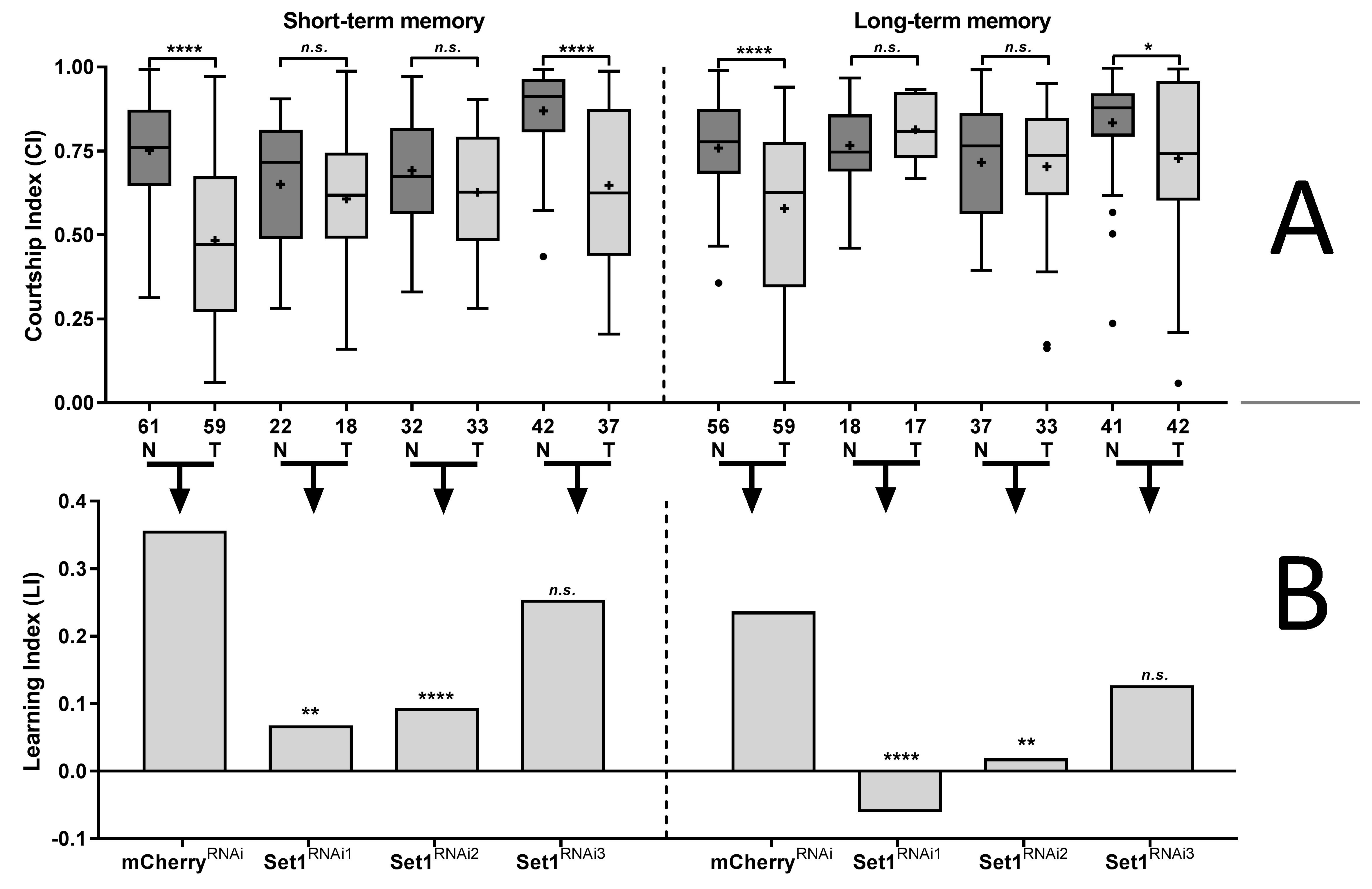


Table 1. Summary of clinical features found in the cohort of 15 individuals with SETD1A variants.*

Frequency Precentage

\begin{tabular}{|c|c|c|}
\hline \multicolumn{3}{|l|}{ Growth } \\
\hline Short stature & $3 / 15$ & $20 \%$ \\
\hline Obesity & $3 / 15$ & $20 \%$ \\
\hline Macrocephaly & $3 / 13$ & $23 \%$ \\
\hline \multicolumn{3}{|l|}{ Development } \\
\hline ID/DD & $14 / 15$ & $93 \%$ \\
\hline Speech delay & $14 / 14$ & $100 \%$ \\
\hline Motor delay & $13 / 14$ & $93 \%$ \\
\hline Behavioural/psychiatric abnormalities & $14 / 15$ & $93 \%$ \\
\hline Sleep disturbance & $7 / 12$ & $58 \%$ \\
\hline Aggressive behavior & $5 / 14$ & $36 \%$ \\
\hline Short attention span & $5 / 14$ & $36 \%$ \\
\hline Anxiety & $4 / 14$ & $29 \%$ \\
\hline Autistic behavior & $3 / 14$ & $21 \%$ \\
\hline \multicolumn{3}{|l|}{ Neurologic abnormalities } \\
\hline Hypotonia & $9 / 15$ & $60 \%$ \\
\hline Seizures & $3 / 15$ & $20 \%$ \\
\hline Structural brain abnormalities on MRI & $4 / 12$ & $33 \%$ \\
\hline \multicolumn{3}{|l|}{ Musculoskeletal abnormalities } \\
\hline Joint hypermobility & $7 / 13$ & $54 \%$ \\
\hline Craniosynostosis & $2 / 15$ & $13 \%$ \\
\hline Gastrointestinal abnormalities & $8 / 12$ & $67 \%$ \\
\hline Feeding difficulties & $3 / 8$ & $38 \%$ \\
\hline Constipation & $3 / 8$ & $38 \%$ \\
\hline Craniofacial dysmorphisms & $15 / 15$ & $100 \%$ \\
\hline \multicolumn{3}{|l|}{ Other } \\
\hline Recurrent infections & $7 / 15$ & $47 \%$ \\
\hline Skin abnormalities & $4 / 15$ & $27 \%$ \\
\hline Visual impairment & $6 / 15$ & $40 \%$ \\
\hline Hearing impairment & $2 / 15$ & $13 \%$ \\
\hline
\end{tabular}

* More extensive clinical information per individual is provided in Supplementary Table 1. As information o 

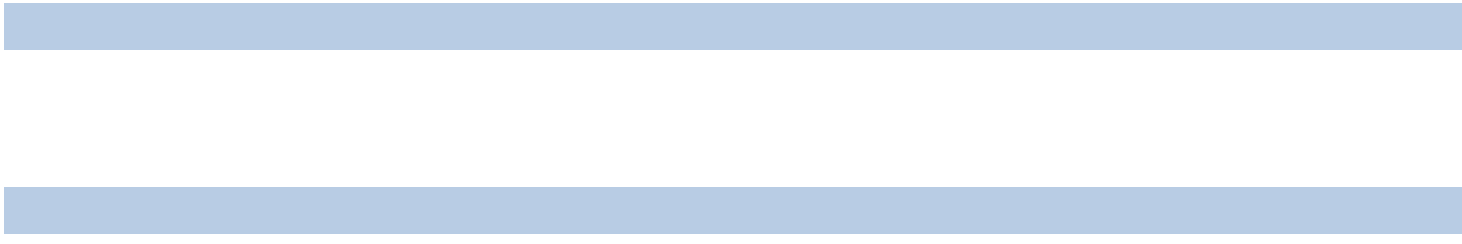

$\mathrm{n}$ the different features was not always applicable or known for each patient, the denominator in the ' $\mathrm{F}$ 

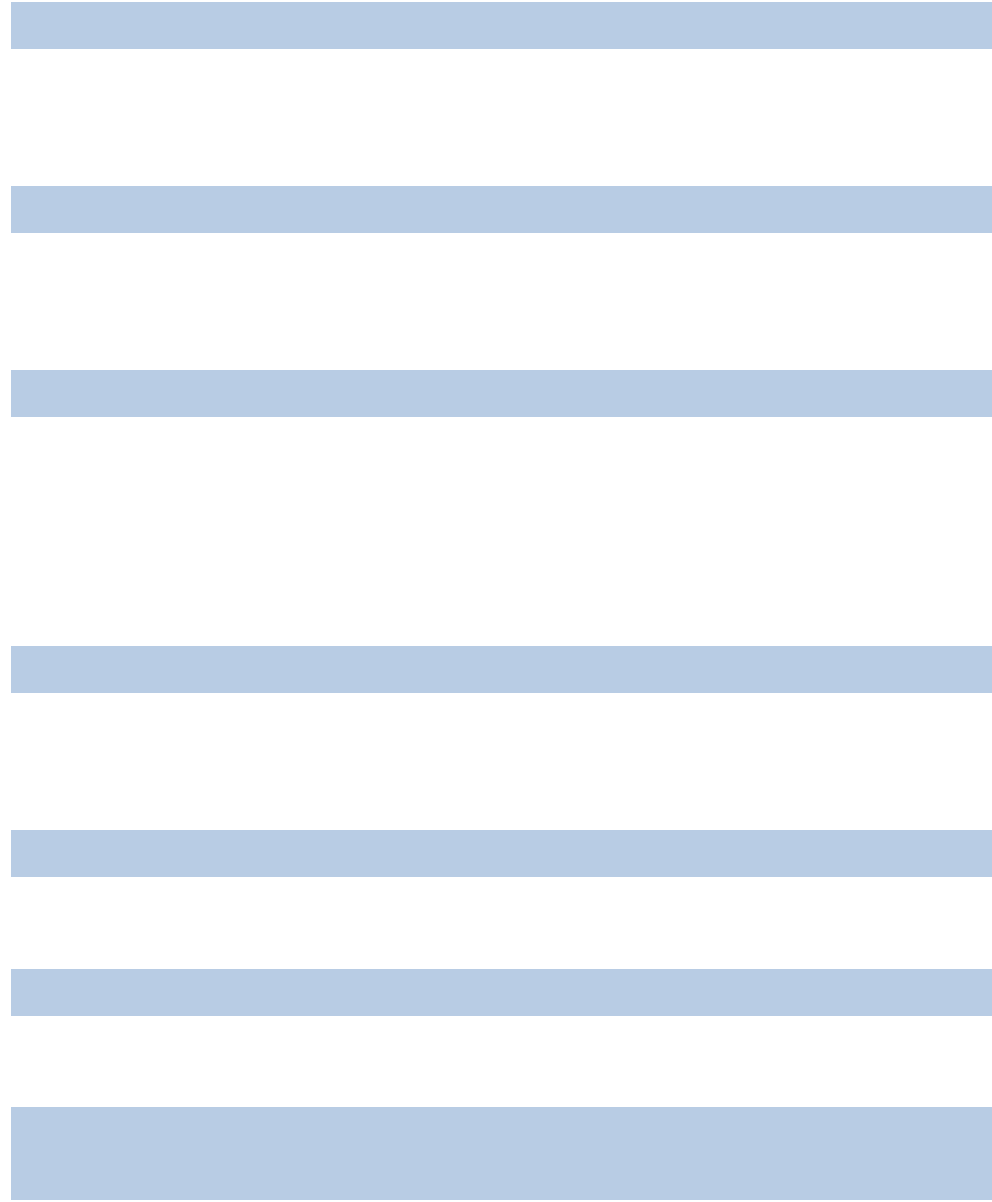

requency' column is different for different clinical characteristics. 